\title{
Sinks Mobility Strategy in IPv6-based WSNs for Network Lifetime Improvement
}

\author{
Leila Ben Saad ${ }^{* \ddagger}$, Bernard Tourancheau ${ }^{\dagger \ddagger}$ \\ * ENS Lyon, INRIA \\ † Université de Lyon, INRIA \\ $\ddagger$ INSA-Lyon, CITI, F-69621, Villeurbanne \\ \{leila.ben.saad, bernard.tourancheau\}@ens-lyon.fr
}

\begin{abstract}
This paper investigates the sinks mobility in IPv6based wireless sensors networks and specially in the new IETF proposed protocol RPL (Routing Protocol for Low power and Lossy Networks). We also show that even the mobility of sinks is not an explicit design criteria, the use of mobile sinks improves the network lifetime. In this work, we propose a new distributed and weighted moving strategy for sinks in RPL. We compared our proposed mobility approach with different others strategies. The results show that our proposed mobility approach notably balances the network load which leads to a significant network lifetime gain in large scale network.
\end{abstract}

Keywords-IPv6 based-WSNs, RPL, Mobile sinks, Network lifetime.

\section{INTRODUCTION}

Recently, significant studies have been made to enable the convergence of sensor networks with IP world and the connectivity of the smart objects to Internet. In fact, The standardization of IEEE 802.15.4[1] provided devices with low cost, low power, low data rate and low bandwidth. The IETF Working Group 6LoWPAN (IPv6 over Low power Wireless Personal Area Networks) proposed the RFC 4944[2] to enable IPv6 packets to be carried over IEEE 802.15.4. The IETF Working Group ROLL (Routing Over Low power and Lossy networks) is currently designing a routing protocol named RPL[3] (IPv6 Routing Protocol for Low power and lossy networks). This protocol targets IPv6-based wireless sensor networks with thousands of sensors and supports a variety of applications including industrial, urban, commercial, home buildings, etc. RPL builds and maintains Directed Acyclic Graphs (DAGs) topologies rooted towards one or many sinks (DAG ROOTs). The data transmitted by the sensors follow only the DAG links.

Designing a routing protocol for multi-hop IP-based WSNs and specially those that are characterized by many-to-one traffic patterns is a very challenging problem. In fact, such networks are formed by a large number of tiny batteryoperated sensors which have a limited and non-renewable energy supply. Furthermore, the sensors behave at the same time as source nodes by generating data to be transmitted to the sinks and as relay nodes by forwarding the data of nodes farther away. Thus, the sensors near the sinks are more likely to use up their energy much faster than distant nodes because they carry heavy traffic. Therefore, the hot spot problem occurs and thus prevents farther nodes to relay their data to the sinks. Consequently, areas of the network are left completely unmonitored and the network lifetime ends prematurely. Although the sinks mobility in RPL is not an explicit design criteria, moving the sinks even infrequently can mitigate this hot-spot problem and increase the network lifetime. Nevertheless, the mobility requires careful handling to limit the overhead of the topology management and ensure that the increased control traffic can not reduce the energy saving obtained by sinks mobility.

The sinks mobility exists in many realistic applications. An example for this can be sensors deployed inside buildings where they are expected to operate for several years without replacing or recharging their batteries. In this case, it may be preferable to simply relocate the sinks or virtually moving them by switching them on or off.

In this paper, we propose a distributed and weighted strategy which improves the performance of RPL in terms of network lifetime by moving the sinks towards the leaf nodes. The simulation results demonstrate that by introducing the sinks mobility in RPL according to our proposed approach, the energy consumption is more balanced among the sensors which leads to a significant increase of network lifetime. To the best of our knowledge, our work is the first to investigate the sinks mobility in RPL for IPv6-based wireless sensor networks.

The rest of this paper is organized as follows. In Section 2, a related work on sinks mobility in WSNs is reviewed. In Section 3, the network model including the major assumptions is described. In Section 4, a brief presentation of RPL protocol is provided. Section 5 discusses the integration of sinks mobility in RPL and explains our proposed moving strategy to improve the network lifetime. Section 6 evaluates the performance of the proposed approach and presents the simulation results. Section 7 concludes the paper.

\section{RELATED WORK}

The lifetime of WSNs can be extended by moving the sinks. Several sink mobility strategies exist in the literature and can be classified into three categories. Mobile sink may move in a fixed path [4], may take a random path [5] or may move in optimal locations in terms of network lifetime or energy conservation. Most of works based on sink mobility suggest to 
move a single sink to improve the network lifetime [6][7][8]. But, few studies focused on the mobility of multiple sinks. To find the optimal placement of multiple mobile sinks, some works formulated the problem as an Integer Linear Program ILP [9][10][11] or Linear Program LP [12]. However, the drawback of LP based solutions is that they are centralized and hard to compute in networks with thousands of sensors due to their high resolution complexity. Another way to determine the positions of mobile sinks in the third category is to use algorithms. In [13], the algorithm, based on iterative clustering, minimizes the distance between sensors and closest sinks. In [14], the algorithm selects the locations of sinks in the periphery of the network in such way that the difference between the maximum and the minimum residual energy of nodes is minimized. In [15], the algorithm moves the sinks towards the distant nodes which have the highest number of hops to reach the nearest sink. Other algorithms make a moving decision according to the complete knowledge of the energy distribution of the sensors. In [16][17], the sinks move towards the zones of nodes with highest residual energy.

The majority of proposed moving strategies are centralized or assume the availability of global information (i.e, residual energy, the location of all sensors). The knowledge of all network parameters is energy-consuming because it needs to be collected which leads to non scalability of these strategies in large scale networks. Contrary to previous works, this paper is aimed to focus on a totally distributed and autonomous strategy without an explicit global network knowledge in large scale IPv6-based WSNs using RPL as protocol.

\section{NETWORK MODEL}

We consider a wireless sensor network composed of static sensor nodes and mobile sinks. We made the following assumptions for the network model.

- We assume $N$ sensors distributed in a bi-dimensional grid network.

- The sensors have a limited initial energy $e_{0}(\mathrm{~J})$ and a fixed transmission range $r(\mathrm{~m})$ equal to the distance between two nodes (i.e, cell size).

- The sinks are moving in the network (really or virtually) from one node to another one.

- The sinks stay at a certain position for at least a certain duration of time. At the end of this duration, they can change their locations.

- A time-driven sampling application of data collection is considered

- Each sensor regularly generates the same amount of data and no aggregation of data is made.

- The network lifetime is defined as the time until the first sensor dies (i.e, it uses up its residual energy). Despite the fact that there are many definitions of the network lifetime, the most used is this one because the death of a node is soon followed by the death of its neighbors.

- Since the energy spent in the communication is the most dominant, only the energy consumption for transmitting and receiving data were considered.
- The protocol used by the sensors to transmit their data to the sinks is the routing protocol RPL.

\section{RPL: IPV6 RouTING PROTOCOL FOR LOW POWER AND LOSSY NETWORKS}

RPL[3] is a routing protocol designed for low power and lossy networks and targets IPv6 based wireless sensor networks with thousands of constrained resources nodes. The basic idea of RPL is that the nodes organize themselves by forming a Destination Oriented DAGs (DODAGs) rooted towards one sink (DAG ROOT) identified by an unique identifier DODAGID. The DODAGs are optimized according to an objective function identified by an Objective Code Point (OCP) which indicates the constraints and the metrics in use (e.g, hop count, latency, expected transmission count, energy). Each node is assigned a rank which determines its relative position in the DODAG. The rank increases down et decreases up. RPL is based on the concept of DAG INSTANCE which is a set of multiple DODAGs. A node can be a member of multiple DAG INSTANCEs but can belong to at most one DODAG per DAG INSTANCE.

RPL constructs and maintains the upwards routes of the DODAGs by the transmission of DODAG Information Object (DIO) messages. DIO messages contains several information (e.g., RPL INSTANCE, DODAGID, RANK, DODAGVersionNumber). The transmission of DIO messages by a node is regulated by a trickle timer[18] to suppress redundant control messages. Each node monitors DIO messages of its neighbors before to join a DODAG. Then, it selects a DODAG parent set from its neighbors according to the cost they advertise and eventually computes its own RANK. Destination Advertisement Object (DAO) messages are aimed to maintain downward routes and they are only used for applications that require point-to-multipoint and point-to-point traffic. Sending a packet to the DAG ROOT consists in selecting the preferred parent with lower rank. To repair the topology of the DODAG and allow nodes to join a new position, the DODAG ROOT increments the DODAGVersionNumber to create a new DODAGVersion. This operation is called global DAG repair.

\section{Sinks Mobility IN RPL}

The main goal of our work is to focus on how to integrate sinks mobility in RPL to improve the lifetime of IPv6-based WSNs characterized by many-to-one traffic patterns. Random sink mobility is not possible in practice and may threaten the network lifetime. The fixed path mobility lacks of flexibility. Autonomous mobility when the sinks take decision according the circumstances at that time is more beneficial to balancing energy. That is why, we focus on a totally distributed and autonomous moving strategy of sinks in RPL.

The lifetime $T$ of a sensor node depends on the energy available at the sensor $\left(e_{0}\right)$ and the energy consumption of the node at each time $\left(e_{c}\right)$ [19].

$$
T=\frac{e_{0}}{e_{c}}
$$




$$
e_{c}=f_{i}\left(e_{T}+e_{R}\right)
$$

Where $f_{i}$ is the average number of packets flowing out from the sensor node $i, e_{T}$ and $e_{R}$ are respectively the unit of energy consumption for transmitting and receiving one packet in each time. This means that the more the sensor has available energy, the longer its lifetime is.

Furthermore to extend the network lifetime, the energy consumption of the whole network should be minimized. In multi-hop networks, the less the number of hops between the sensor and the sink is, the less the energy consumption is. In fact, the energy cost of transmitting a packet to the $\operatorname{sink}\left(E_{p k t}\right)$ is linearly proportional to the number of hops the packet has to travel denoted here as $h$ [13]. Let $E_{1}$ be the energy cost to send a packet in 1-hop distance.

$$
E_{p k t} \sim h E_{1}
$$

Therefore to increase the lifetime, the number of hops between the sensors and the sink should be reduced. However, the number of hops depends on the routing protocol, the sensor nodes configuration, density and the sinks positions. The manipulation of the sensors density and configuration is not always possible. Therefore one way to reduce the number of hops without changing the protocol is to optimize the configuration of sinks by moving them. In addition to the sensor energy and the number of hops, the number of neighbors is another parameter influencing the network lifetime. In fact, the more the sinks have neighbors, the more the traffic load is balanced among nodes and the longer network lifetime is.

Our proposed moving strategy (RPL_Weight) relies on these three parameters which are locally available at each sensor node. Notice that in RPL each node can determine its number of hops to reach the sink from the DIO messages advertised by its parents. Let $h_{i}^{k}$ be the number of hops from sensor node $i$ to its DAG ROOT at position $k, e_{i}$ be the residual energy of sensor node $i$ and $b_{i}$ be the number of its 1-hop neighbors. For each leaf node of the DODAG, we define a weight $w_{i}$ which is a function of the parameters $h_{i}^{k}, e_{i}$ and $b_{i}$.

$$
w_{i}=f\left(h_{i}^{k} e_{i} b_{i}\right)
$$

The exact calculation of the weight is as follows.

$$
w_{i}=\beta h_{i}^{k} e_{i}+\gamma b_{i}
$$

$\beta$ and $\gamma$ are coefficients of normalization. They are introduced to mitigate the effect of scale since the units of measurement are different.

Moving the sinks really or virtually in RPL involves a change of the address assigned to the sink node (i.e., DODAGID). Therefore, such mobility can be seen as the case of having many DAG INSTANCEs containing different DAG ROOTs. In order to make the sinks mobility possible in RPL, we define the period $T_{\text {instance }}$ which is the period of creation of a new instance and corresponds to the moment when the sinks are allowed to move in a new position among the nodes.
The period $T_{\text {instance }}$ is a multiple of the period of the DAG repair denoted here as $T_{\text {version }}$ to mitigate the overhead of the control messages generated by the sinks mobility.

$$
T_{\text {instance }}=p T_{\text {version }} \quad p \in N^{*}
$$

Where $p$ is the instance period multiplier.

The process of sinks relocation needs three phases:

1) During the $T_{\text {instance }}$ period, each node monitors the RANKs advertised by its neighbors belonging to the same DODAG. When, it does not hear a node with the rank higher than itself, it considers itself as a leaf node.

2) Before the end of $T_{\text {instance }}$, each leaf node sends Data packet by piggybacking its weight $w_{i}$.

3 ) At the beginning of a new $T_{\text {instance }}$, each DAGROOT determines the leaf node with highest weight and move there.

\section{Simulation Results}

Simulations were performed on the WSnet simulator [20]. A basic version of RPL with three mobile sinks was implemented. The Chipcon radio $\mathrm{CC} 1100$ with $250 \mathrm{kbps}$ data rate and the IEEE 802.15.4 MAC and PHY layer specifications were used. The current consumption values in transmit and receive mode were respectively chosen equal to $16.9 \mathrm{~mA}$ and $16.4 \mathrm{~mA}[21]$. The sensor transmission range $r$ was fixed to $10 \mathrm{~m}$. All the data packets generated by the sensors were of size 127 bytes and were destined to the DAG ROOTs. The packets were sent every one minute according to a Constant Bit Rate (CBR). The hop count was used as a metric to build the DODAGs. The trickle time interval for emitting DIO messages was initially fixed to 1 second and then changed exponentially over the simulation time as specified in [18]. Since the traffic supported in the application is convergecast to the sink, only upwards routes were considered and the DAO messages advertisement was configured to be entirely disabled.

To show the benefit of sinks mobility, we evaluated the performance of RPL by making a comparative study with different mobility strategies:

1) RPL_Static

2) RPL_Random

3) RPL_Energy

4) RPL_Weight

5) RPL_ILP

In strategy (1), the sinks are static. In strategy (2), the sinks are moving randomly among the sensor nodes. In strategy (3), the sinks are moving towards the nodes with the highest energy. The strategy (4) is our proposed approach as presented in Section V. The sinks are moving towards the leaf node with the highest weight. In strategy (5), the sinks are moving according to the optimal solution computed with an Integer Linear Program detailed in [11]. This latter strategy will be used only in small networks because its is hard to apply it in large scale network due to the high resolution complexity.

In the following sections, we focus on the performance of RPL with mobile sinks by analyzing the network lifetime, the residual energy and the amount of control messages. 
TABLE I

NETWORK LIFETIME GAP RELATIVELY COMPARED TO ILP SOLUTION

\begin{tabular}{|l|c|c|}
\hline Strategy & $4 \times 4$ sensors & $5 \times 5$ sensors \\
\hline RPL_Static & $44.46 \%$ & $45.20 \%$ \\
\hline RPL_Energy & $31.80 \%$ & $34.69 \%$ \\
\hline RPL_Random & $28.75 \%$ & $33.26 \%$ \\
\hline RPL_Weight & $22.00 \%$ & $25.50 \%$ \\
\hline
\end{tabular}

\section{A. The network lifetime}

We simulated RPL and observed the effect of sinks mobility on the network lifetime.

Table I shows the percentage of the lifetime gap of the moving strategies with respect to the optimal solution computed by the ILP in small scale networks. Our proposed strategy RPL_Weight achieves the closest network lifetime to the optimum comparing to other strategies. The gap of our proposed approach is about $22 \%$ and $25.5 \%$ respectively in networks with 16 and 25 nodes. This gap can be considered as acceptable since the proposed mobility strategy is totally distributed and does not need a global information of all sensor nodes.

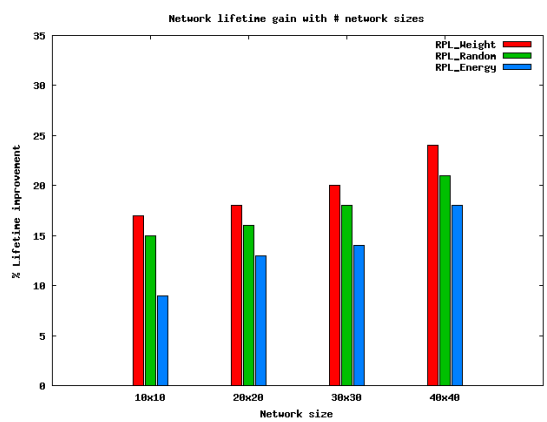

Fig. 1. Lifetime improvement with different network sizes

Figure 1 compares the lifetime gain achieved by the different moving strategies with respect to RPL_Static strategy when varying the network size. The results shows that the lifetime improvement increases with the size of the network. This straightforwardly proves that using mobile sinks is more beneficial in large scale networks. It is also observable that the lifetime gain obtained by our proposed strategy is better than the other strategies independently of the size of the network. Moreover, the lifetime improvement induced by our approach is about $24 \%$ in network with 1600 nodes.

Figure 2 displays the lifetime improvement achieved by the moving strategies with respect to RPL_Static strategy for different values of initial energy of the sensors in network with 1600 nodes. As expected, the lifetime gain is always higher than the others approaches and varies between 23 to $25 \%$.

Figure 3 shows the effect of increasing the period $T_{\text {instance }}$ and varying the period of DAG repair on network lifetime of the two strategies RPL_Weight and RPL_Static. When the period $T_{\text {instance }}$ increases, the network lifetime of RPL_weight decreases slightly. This can be explained by the fact that long sojourn time of sinks at certain locations causes less sinks

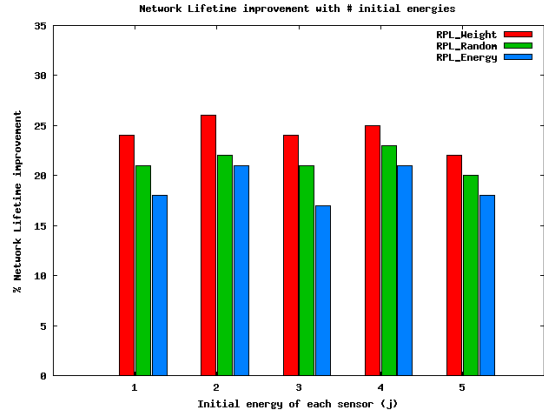

Fig. 2. Lifetime improvement with different initial energies of sensors

movements. So, the sensors near the sinks consume more energy which leads to shorter lifetime. We notice also that short periods of DAG repair have an influence on network lifetime because they generate higher rate of control packets which minimizes the network lifetime.

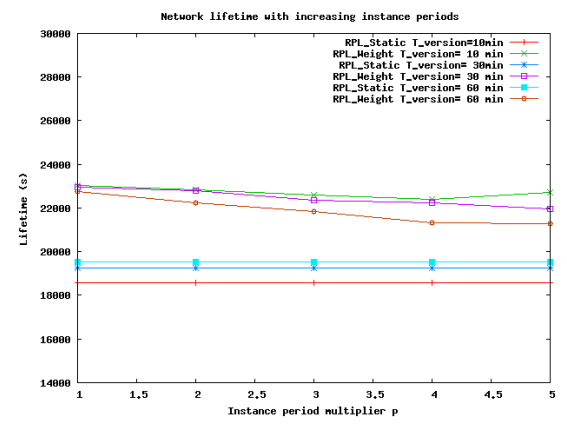

Fig. 3. Lifetime with different periods of $T$ _instance

\section{B. The residual energy}

Figure 4 shows the percentage of sensors residual energy in various networks size at network lifetime end.

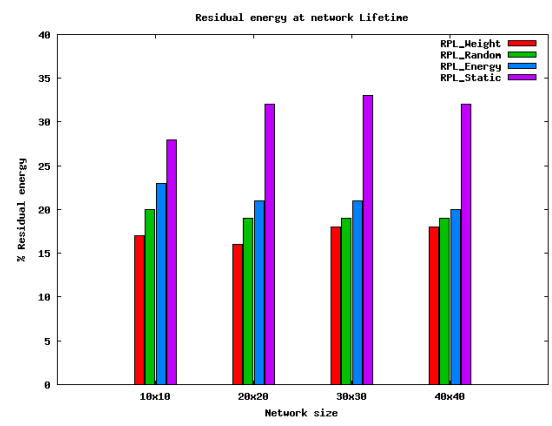

Fig. 4. Residual energy at network lifetime end

The percentage of energy left unused at the end of network lifetime in mobile sinks strategies is notably lower than in the case of static sinks. This is due to the fact that the sinks mobility changes the nodes acting as relays frequently and leads to balanced energy consumption among nodes. Nevertheless, our proposed strategy results in the best distribution of the 
available energy on the sensors since it leaves the smallest amount of unused energy at the end of network lifetime.

\section{The packets overhead}

In the Figure 5, we analyzed the amount of data packets transmitted (including forwarded data packets) and the ICMPv6 control packets (DIO messages) transmitted by each node. When the sinks are static, the nodes near the sinks (e.g, node id 778) have more data traffic than others nodes because they have to transmit their own data in addition to data of nodes farther away. Concerning leaf nodes (e.g, node id 1401), the amount of data packets transmitted is smaller than that of nodes in the middle or close to the sink. This is because they do not have to act as forwarding nodes. By moving the sinks according to our weighted approach, the nodes playing the role of relay nodes change and the data traffic becomes more balanced among all the nodes. As shown in the Figure 5, the majority of nodes have a comparable amount of data packets transmission. Moreover, the control overhead is very small in comparison to data packets. It is also not highly increased in spite of the mobility of sinks. This can be explained by the fact that the sinks move only during the periods of DAG repair.

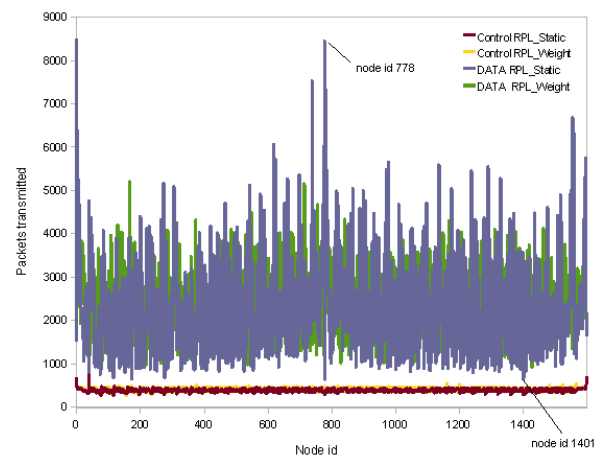

Fig. 5. Packets transmitted : Control packets and Data packets (including forwarded data)

\section{CONCLUSION}

In this paper, we investigated the sinks mobility in the new protocol RPL. We also proposed an autonomous and distributed moving strategy to extend the lifetime of large scale IPv6-based WSNs. Our proposed approach moves the sinks towards the nodes with the highest weight. This weight is a function of three parameters influencing the network lifetime: residual energy, number of neighbors and number of hops. This approach does not need a global knowledge and prevents from collecting information from all the sensors since it uses only leaf nodes of the DODAGs. Furthermore, to mitigate the overhead of the control messages, the sinks are allowed to change their location only during periods of global DAG repair. We evaluated the performance of our proposed strategy by simulation in networks with thousands of sensors and compared it with others strategies. The results show that it extends significantly the network lifetime. The gain achieved in a network with 1600 sensors and 3 mobile sinks is about
$24 \%$ with the respect to the static strategy. Nonetheless, longer network lifetime can be achieved in RPL if the overhead of DIO messages were less important and better controlled by well choosing the parameters of the trickle timer.

In our future work, we intend to improve the weight function and implement RPL with mobile sinks in real-life deployment topologies. We would like also to study its performance with different traffic patterns using a variety of metrics in DAG construction and mechanisms of DAG repair.

\section{REFERENCES}

[1] IEEE Std. 802.15.4-2003 IEEE Computer Society, October 2003.

[2] G. Montenegro, N. Kushalnagar, J. Hui, and D. Culler, "Transmission of IPv6 packets over IEEE 802.15.4 networks," IETF, Request for Comments 4944, September 2007.

[3] T. Winter and P. Thubert, "RPL: IPv6 routing protocol for low power and lossy networks," IETF, Internet-Draft draft-ietf-roll-rpl-11, July 2010.

[4] J. Luo and J.-P. Hubaux, "Joint mobility and routing for lifetime elongation in wireless sensor networks," In Proceedings 24th Annual Joint Conference of the IEEE Computer and Communications Societies INFOCOM, 2005.

[5] R. C. Shah, S. Roy, S. Jain, and W. Brunette, "Data mules: Modeling a three-tier architecture for sparse sensor networks," in IEEE International Workshop on Sensor Network Protocols and Applications SNPA, 2003, pp. 30-41.

[6] Z. M. Wang, S. Basagni, E. Melachrinoudis, and C. Petrioli, "Exploiting sink mobility for maximizing sensor networks lifetime," Proceedings of the 38th Annual Hawaii International Conference on System Sciences HICSS, 2005.

[7] B. Wang, D. Xie, C. Chen, J. Ma, and S. Cheng, "Employing mobile sink in event-driven wireless sensor networks," IEEE Vehicular Technology Conference VTC, 2008.

[8] K. Akkaya and M. Younis, "Sink repositioning for enhanced performance in wireless sensor networks," Elsevier Computer Networks Journal, 2005.

[9] S. R. Gandham, M. Dawande, R. Prakash, and S. Venkatesan, "Energy efficient schemes for wireless sensor networks with multiple mobile base stations," The IEEE Global Telecommunications Conference GLOBECOM, vol. 1, 2003.

[10] W. Alsalih, S. Akl, and H. Hassanein, "Placement of multiple mobile base stations in wireless sensor networks," IEEE International Symposium on Signal Processing and Information Technology, 2007.

[11] L. Ben Saad and B. Tourancheau, "Towards an optimal positioning of multiple mobile sinks in WSNs for buildings," International Journal On Advances in Intelligent Systems IARIA, 2009.

[12] S. Basagni, A. Carosi, C. Petrioli, and C. Phillips, "Moving multiple sinks through wireless sensor networks for lifetime maximization," in 5th IEEE International Conference on Mobile Ad Hoc and Sensor Systems MASS, 2008.

[13] Z. Vincze, R. Vida, and A. Vidacs, "Deploying multiple sinks in multi-hop wireless sensor networks," IEEE International Conference on Pervasive Services, 2007.

[14] A. Azad and A. Chockalingam, "Mobile base stations placement and energy aware routing in wireless sensor networks," IEEE Wireless Communications and Networking Conference WCNC, 2006.

[15] L. Ben Saad and B. Tourancheau, "Towards an efficient positioning of mobile sinks in wireless sensor networks inside buildings," in WSN theory and practice Workshop in conjunction with NTMS, 2009.

[16] M. Marta and M. Cardei, "Improved sensor network lifetime with multiple mobile sinks," Pervasive and Mobile computing, 2009.

[17] Y. Bi, J. Niu, L. Sun, W. Huangfu, and Y. Sun, "Moving schemes for mobile sinks in wireless sensor networks," Performance, Computing, and Communications Conference, 2007.

[18] P. Levis, T. Clausen, J. Hui, O. Gnawali, and J. Ko, "The trickle algorithm," IETF, draft-ietf-roll-trickle-03, August 2010.

[19] W. Wang, V. Srinivasan, and K.-C. Chua, "Using mobile relays to prolong the lifetime of wireless sensor networks," in MobiCom '05: Proceedings of the 11th annual international conference on Mobile computing and networking. ACM, 2005, pp. 270-283.

[20] WSnet simulator http://wsnet.gforge.inria.fr/.

[21] CC1100 datasheet http://focus.ti.com/lit/ds/symlink/cc1100.pdf. 